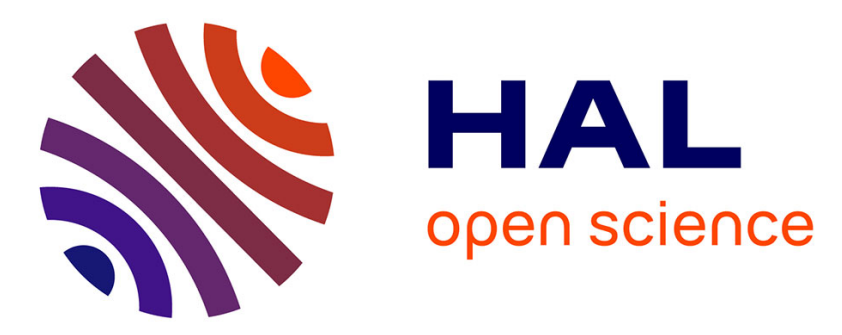

\title{
Formes graves de paludisme à Plasmodium vivax: caractéristiques sociologiques, cliniques et biologiques associées à l'anémie profonde
}

Leila Baha, Denis Blanchet, Narcisse Elenga, Gérald Egmann, Bernard Carme, Félix Djossou, Magalie Pierre Demar, Loïc Epelboin

\section{To cite this version:}

Leila Baha, Denis Blanchet, Narcisse Elenga, Gérald Egmann, Bernard Carme, et al.. Formes graves de paludisme à Plasmodium vivax: caractéristiques sociologiques, cliniques et biologiques associées à l'anémie profonde. 18 ème Journées Nationales d'Infectiologie, Jun 2017, Saint-Malo, France. hal02870481

\section{HAL Id: hal-02870481 https://hal.univ-guyane.fr/hal-02870481}

Submitted on 16 Jun 2020

HAL is a multi-disciplinary open access archive for the deposit and dissemination of scientific research documents, whether they are published or not. The documents may come from teaching and research institutions in France or abroad, or from public or private research centers.
L'archive ouverte pluridisciplinaire HAL, est destinée au dépôt et à la diffusion de documents scientifiques de niveau recherche, publiés ou non, émanant des établissements d'enseignement et de recherche français ou étrangers, des laboratoires publics ou privés. 


\section{MIG-10}
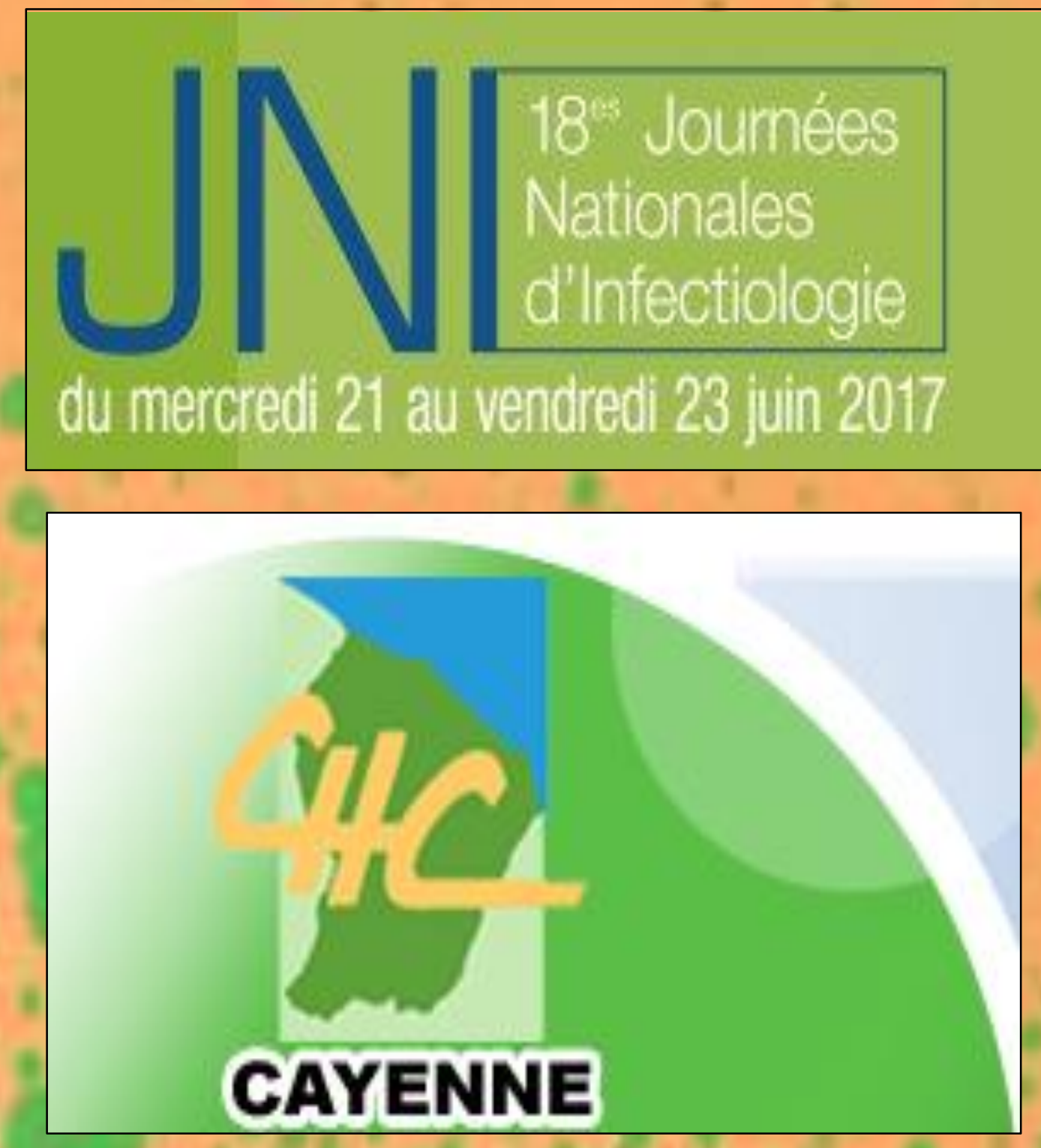

\section{Formes graves de paludisme à Plasmodium} vivax : caractéristiques sociologiques, cliniques et biologiques associées à l'anémie profonde

Leila Baha ${ }^{1}$, Denis Blanchet ${ }^{1,2}$, Narcisse Elenga ${ }^{1,2}$, Gérald Egmann ${ }^{1,2}$, Bernard

Carme $^{1,2}$, Félix Djossou ${ }^{1,2}$, Magalie Demar ${ }^{1,2}$, Loïc Epelboin ${ }^{1,2}$

1. Centre Hospitalier Andrée Rosemon, F-97300 Cayenne, French Guiana

2. Equipe EA 3593, Ecosystèmes Amazoniens et Pathologie Tropicale, Université de Guyane, F-97300 Cayenne, French Guiana

\section{INJRODUGTION}

Plasmodium falciparum $(P f)$ et $P$. vivax $(P v)$ :espèces de paludisme les plus répandus en Amérique latine, respectivement $25 \%$ et $75 \%$

- $P f=$ potentiellement mortel, avec des critères de gravité définis par I'OMS, et la SPILF

- $P V=$ réputation de bénignité sans forme grave mais avec récurrences Formes graves de Pv, surtout décrites en Asie, peu décrites en Amérique latine; Critère de gravité le plus souvent retrouvé = anémie profonde Objectifs de l'étude

- déterminer la fréquence de l'anémie profonde au cours du paludisme à Pv

- rechercher les facteurs prédictifs et caractéristiques sociologiques, cliniques et biologiques des patients avec anémie profonde au cours d'une infection à Pv

\section{PAJJENTS ETMETHODES}

Etude observationnelle, rétrospective, monocentrique, cas-témoin appariée

Population d'étude : patients pris en charge entre 01/01/2007 et le 31/12/2014 au CH de Cayenne

Critères d'inclusion :

- frottis-goutte épaisse positif à P. vivax

- taux d'hémoglobine $(\mathrm{Hb})<7 \mathrm{~g} / \mathrm{dl}$ ou hématocrite $(\mathrm{Hte})<20 \%$ chez l'adulte et $\mathrm{Hb}<5 \mathrm{~g} / \mathrm{dl}$ ou $<15 \%$ respectivement chez l'enfant $<15$ ans à \pm 3 jours du diagnostic microbiologique de paludisme

Critères d'exclusion : co-infection à P. falciparum ; absence de dossier médical

Groupe témoin : infection à $P$. vivax et $\mathrm{Hb}>12 \mathrm{~g} / \mathrm{dl}$ et apparié sur la date du diagnostic. 2 témoins pour 1 cas.

Analyse bivariée et analyse multivariée : régression logistique pas à pas descendante. Exclusion des variables avec $>10 \%$ de données manquantes; inclusion des variables avec $p<0,2$

\section{RESULTAJS}

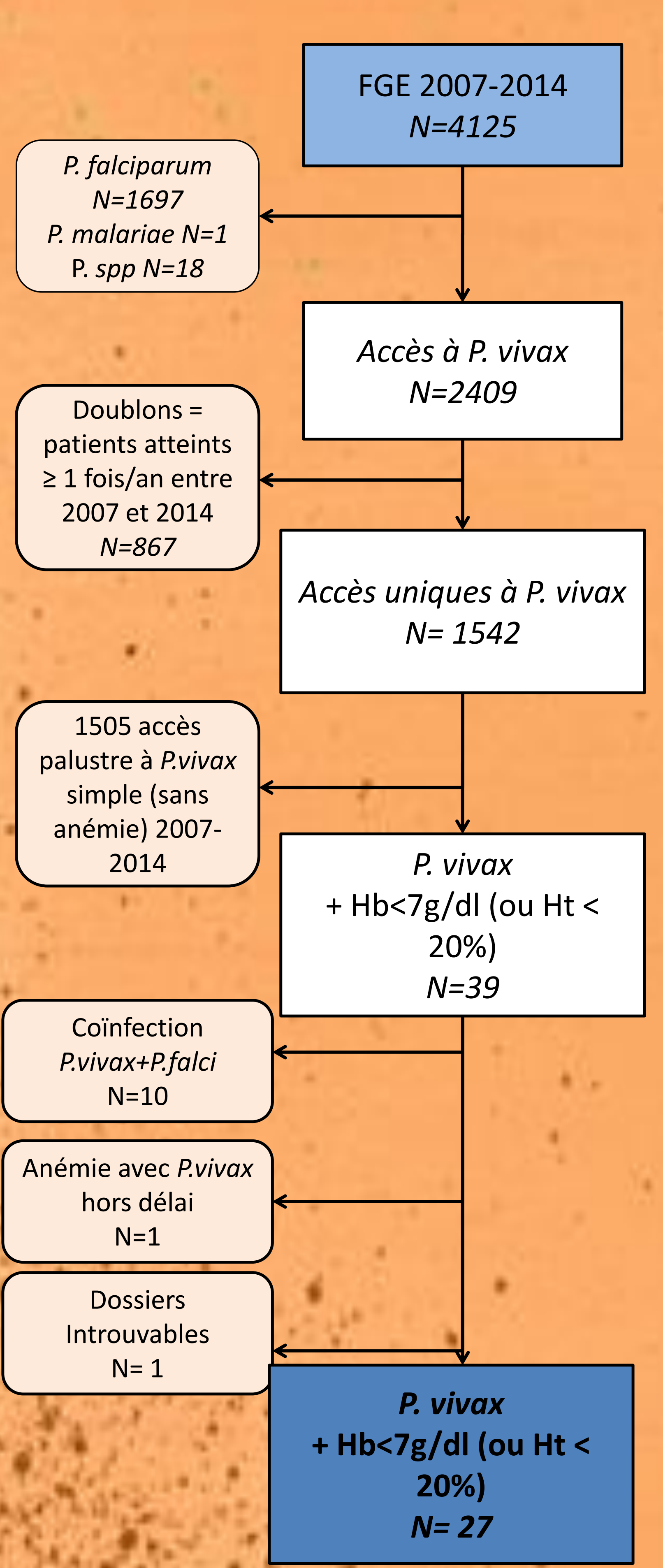

\section{RESULTATS}

1542 accès palustres à $P$. vivax sur 8 ans, 27 avec anémie profonde soit $1,75 \%$ des accès palustres à $P$. vivax

médiane 1,5 cas par an (min-max 1-12 ; Q25Q75 1-3) Signes

\section{Analyse bivariée}

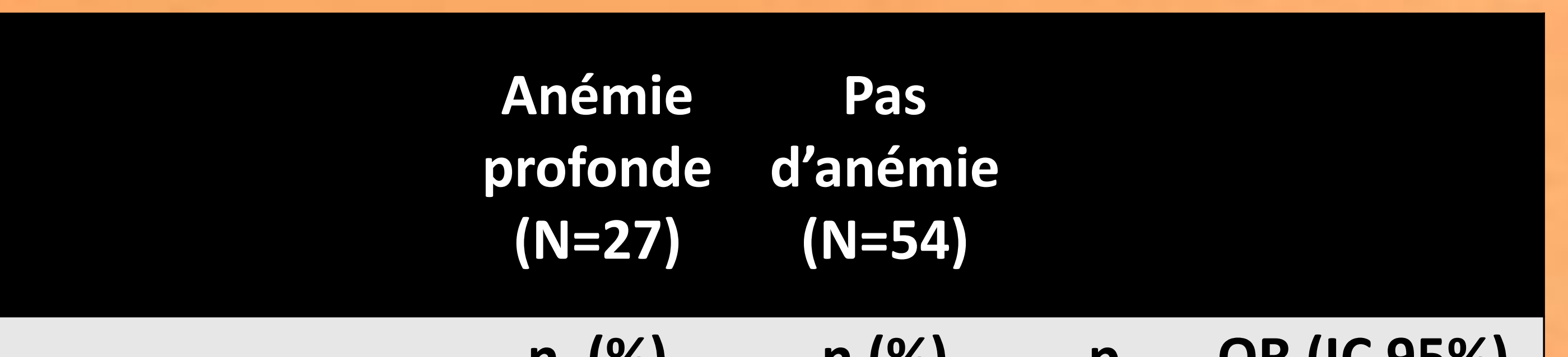

Résidence isolé

fleuve intérieur $10 / 27$ (38) $7 / 54$ (13) $0,042 \quad 4(1,05-15,2)$

Grossesse $\quad 6 / 6(100) \quad 0 / 21(0) \quad 0,004>5 e+16$ (NR)

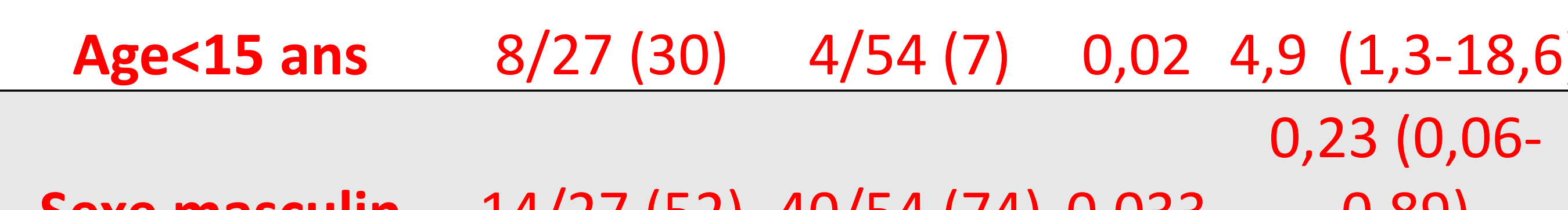

Durée fièvre >7j $13 / 21$ (62) $11 / 52$ (21) 0,007 8,1 (1,8-37,8)

\begin{tabular}{ccccc} 
hémorragiques $\quad 8 / 27(30)$ & $4 / 54(7)$ & $0,024 \quad 4(1,2-13,3)$ \\
\hline & & & $7,6(0,89-$
\end{tabular}

\begin{tabular}{ccccc} 
Splénomégalie & $11 / 16(69)$ & $5 / 30(17)$ & 0,063 & $63,9)$ \\
\hline Ictère & $3 / 9(33)$ & $2 / 13(15)$ & 0,19 & \\
\hline
\end{tabular}

Signes de gravité

autres qu’anémie 14/27 (52) 6/54 (11) $0,001 \quad 6,3(2,1-19,3)$

$\begin{array}{lllll}\text { Hospitalisation } & 26 / 27(96) & 13 / 53(25) & 1 & >7 e+16\end{array}$ (NR)

\begin{tabular}{|c|c|c|c|c|}
\hline & $\begin{array}{c}\text { Anémie } \\
\text { profonde } \\
(\mathrm{N}=27)\end{array}$ & $\begin{array}{c}\text { Pas } \\
\text { d'anémie } \\
(\mathrm{N}=54)\end{array}$ & & \\
\hline & n (\%) & n (\%) & $\mathbf{p}$ & $\begin{array}{l}\text { OR (IC } \\
95 \%)\end{array}$ \\
\hline $\begin{array}{c}\text { Parasitémie } \geq \\
0.00125 \%\end{array}$ & $6 / 27(22)$ & $6 / 53(11)$ & 0,19 & \\
\hline VGM <80 & $9 / 25(36)$ & $3 / 53(6)$ & 0,007 & $\begin{array}{c}8,3(1,8- \\
38,7) \\
\end{array}$ \\
\hline $\begin{array}{c}\text { PNN }<2000 \\
(\text { Vs }>2000)\end{array}$ & $7 / 27(26)$ & $5 / 53(9)$ & 0,068 & $\begin{array}{c}3,2(0,91- \\
11,0)\end{array}$ \\
\hline $\begin{array}{l}\text { Lymphocytes } \\
<500 / \mathrm{mm} 3\end{array}$ & $4 / 26(15)$ & $18 / 52(35)$ & 0,048 & $\begin{array}{c}0,27(0,07- \\
0,99) \\
\end{array}$ \\
\hline plq $<100 \mathrm{G} / \mathrm{L}$ & $\begin{array}{c}11 / 26 \\
(42) \\
\end{array}$ & $36 / 53(68)$ & 0,036 & $\begin{array}{c}0,32(0,11- \\
0,93) \\
\end{array}$ \\
\hline ALAT >40 UI/L & $3 / 22(14)$ & $16 / 49(33)$ & 0,07 & $\begin{array}{c}0,24(0,05- \\
1,13)\end{array}$ \\
\hline Créat>110 $\mu \mathrm{M}$ & $3 / 26(12)$ & $0 / 54(0)$ & 0,01 & \\
\hline $\begin{array}{c}\text { Glycémie<3,3 } \\
\mathrm{mM}\end{array}$ & $3 / 25(12)$ & $0 / 53(0)$ & 0,01 & \\
\hline $\begin{array}{c}\text { Réserve } \\
\text { alcaline }>23 \mathrm{mM}\end{array}$ & $\begin{array}{c}10 / 25 \\
(10)\end{array}$ & $8 / 51(16)$ & 0,027 & $\begin{array}{c}3,8(1,2- \\
12,5)\end{array}$ \\
\hline $\mathrm{Ca}<2,2 \mathrm{mM}$ & $\begin{array}{c}19 / 22 \\
(86)\end{array}$ & $22 / 47$ (47) & 0,01 & $\begin{array}{c}15,3(1,9- \\
120,6)\end{array}$ \\
\hline
\end{tabular}

Analyse multivariée

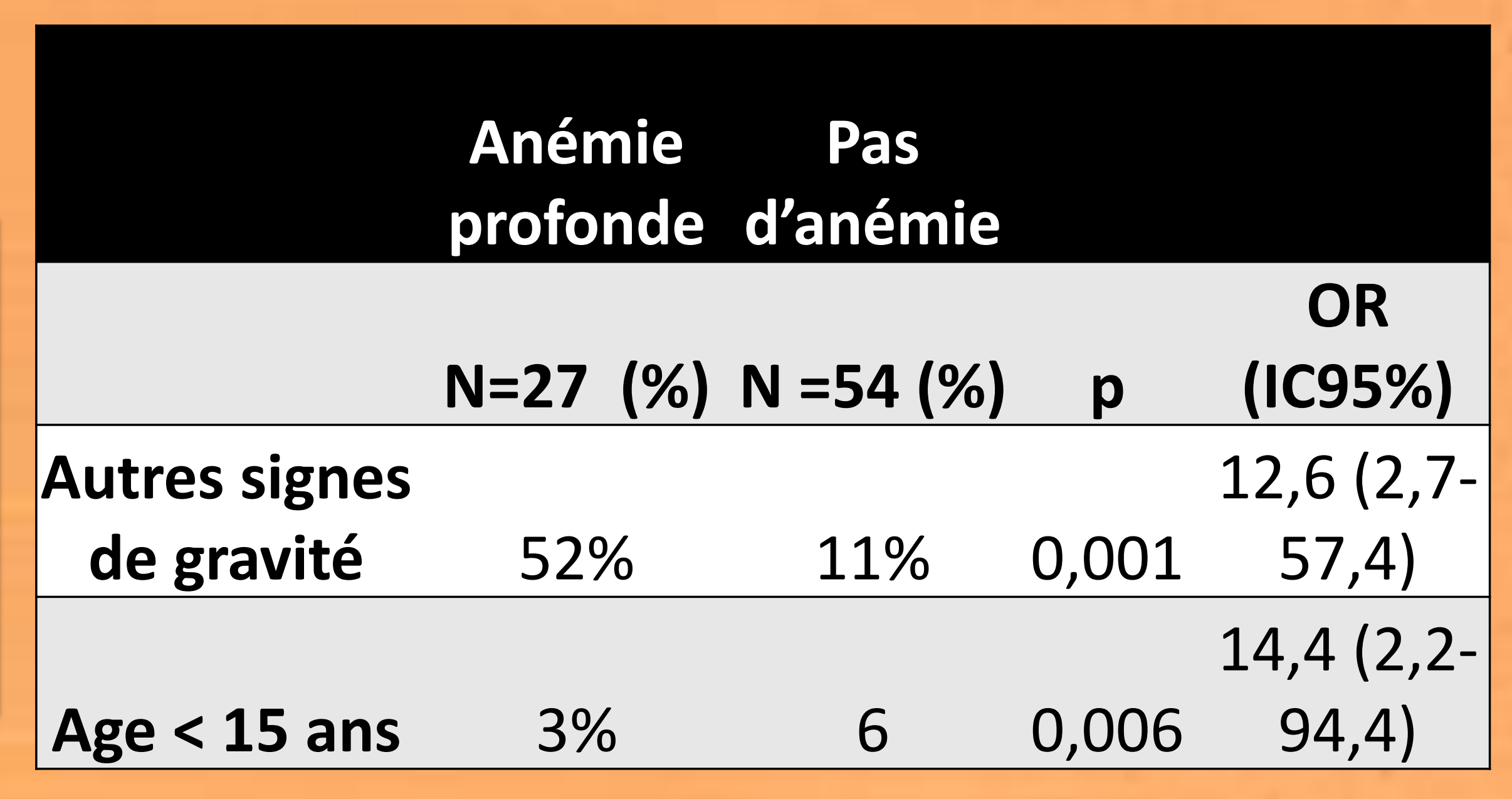

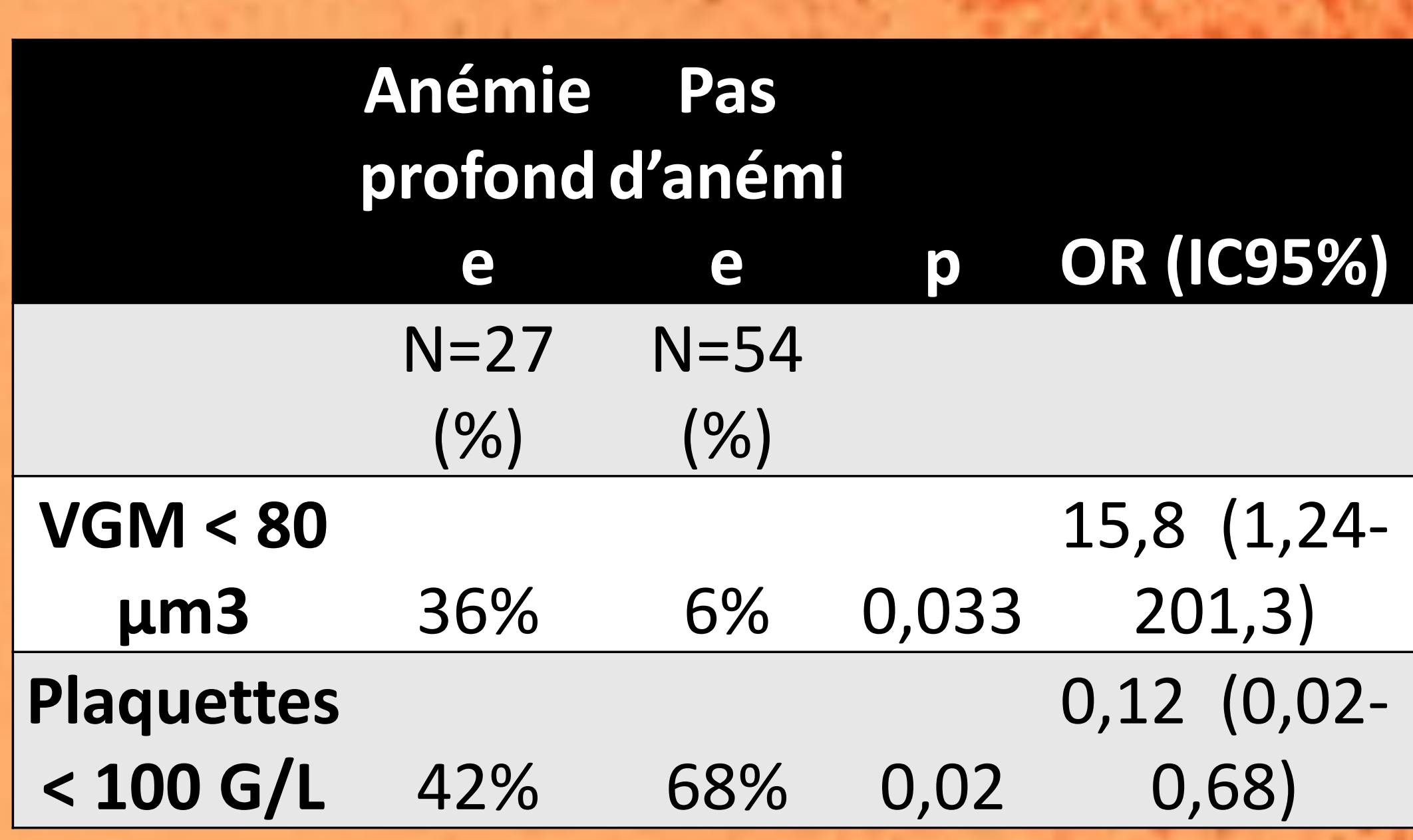

\section{DISCUSSION/CONCLUSION}

Anémie sévère $=$ phénomène rare au cours de l'accès à $P$. vivax en Guyane

Thrombopénie plus rare chez ces patients (stimulation médullaire ?)

2 hypothèses qui s'entrecroisent

$\checkmark$ survenue d'accès palustres simples chez des patients avec terrain prédisposé à l'anémie (grossesse, enfants carencés des communes isolées)

$\checkmark$ existence de forme graves de $\boldsymbol{P}$. vivax, anémie associée alors à d'autres signes de gravité tels que définis par l'OMS et la SPILF. 